\section{Response to Letter: Intrapatient Comparison of the Hepatobiliary Phase of Gd-BOPTA and Gd-EOB-DTPA in the Differentiation of HCA From FNH}

Maarten G. Thomeer, MD, $\mathrm{PhD},{ }^{1 *} \mathbb{C}$

Inge J.S.M.L. Vanhooymissen, $\mathrm{MD},{ }^{1}$

Loes M.M. Braun, MD, PhD, ${ }^{1}$ Sebastiaan van Koeverden, $\mathrm{MD},{ }^{2}$

Francois E. Willemssen, MD, ${ }^{1}$ Robert A. De Man, MD, PhD, ${ }^{3}$

Jan N. Ijzermans, MD, PhD, ${ }^{4}$ and

Roy S. Dwarkasing, MD, $\mathrm{PhD}^{1}$

\section{To the Editor:}

We thank Edouard Reizine et al for their interest in our work and for their thoughtful comments. As mentioned in our article, and in accordance with the current literature, the hepatobiliary phase (HBP) of Gd-BOPTA does not appear to be reliable to differentiate between hepatocellular adenoma (HCA) and focal nodular hyperplasia $(\mathrm{FNH}){ }^{1}$ Several remarks mentioned in the letter will be addressed.

\section{$\mathrm{T}_{1}$-Weighted Hyperintensity Before Contrast Injection}

As suggested by our findings, difficulties concerning HBP may be partially due to precontrast hyperintensity of the lesion. The letter's authors argue that this is probably due to underlying steatosis, which will reveal the lesion as hyperintense on fat-saturated images. We agree with this and have previously highlighted this potential diagnostic dilemma, particularly in inflammatory HCAs after injection of Gd-BOPTA. ${ }^{2}$ However, in contrast to previously published data from Reizine et al, we found that surrounding steatosis is not the only cause of hyperintensity on precontrast images. $^{3}$ Intrinsic hyperintensity is demonstrably an additional source of false-positive iso/hyperintensity in HBP. This can easily be appreciated on in-phase $T_{1}$-weighted images and is probably due to residue from microscopic bleeds. Upon reanalysis of our data, we found that hyperintensity on precontrast images was due to steatosis in $29 / 41$ cases $(71 \%)$, intrinsic hyperintensity in $7 / 41$ cases $(17 \%)$, and was attributable to both causes in $5 / 41$ cases $(12 \%)$. More important, intrinsic hyperintensity is a very reliable sign for HCA, as it is never seen in FNH (because FNHs simply do not bleed). ${ }^{4}$

\section{Enhancement vs. Pseudoenhancement}

We agree with the authors that real uptake versus pseudoenhancement (due to surrounding steatosis or intrinsic hyperintensity) is an important issue when relying on the HBP alone; even more so since uptake is related to glutamine synthetase expression and bcatenin positivity. ${ }^{5}$ Tumors that show noticeable uptake in HBP include FNH, inflammatory HCAs, b-catenin HCAs, and some well-differentiated hepatocellular carcinomas (HCCs). ${ }^{6}$ It should also be noted that in most cases FNH can be easily differentiated from other lesions (which may require closer observation) based on additional radiological and clinical parameters.

\section{LLCER Method}

The LLCER (lesion-to-liver contrast enhancement ratio) method, as proposed by Roux et $\mathrm{al}^{7}$ and mentioned by the authors of the letter, is an elegant approach to objectify whether iso/hyperintensity in HBP is due to uptake of contrast. This method takes into account the intensity of the lesion before contrast injection. Theoretically, if the LLCER is above a certain threshold the uptake is real, thus favoring $\mathrm{FNH}$, or in some rare cases, inflammatory HCA, b-catenin HCA, or HCC. Unfortunately, the benefits of LLCER currently remain within the confines of theory, as we have shown that the method is poorly reproducible, and most important, does not provide reliable cutoffs. ${ }^{8}$ These problems are due to the fact that the patient is removed from the bore after the dynamic phase, to return only after around 1 hour. In contrast to computed tomography (CT), absolute values cannot be determined in magnetic resonance imaging (MRI) and repositioning of the patient has a very disruptive effect on absolute measurements. One way to potentially overcome this problem is by comparing relative measurements before and 1 hour after contrast injection. However, this approach also appears to have limited reliability, based on our previous validation study. ${ }^{8}$ At the present time we are forced to conclude that this method needs further refinement and is therefore not yet suitable for use in daily clinical practice.

\section{Inflammatory HCA}

The final remarks of Reizine et al relate to the fact that the subtyping of inflammatory HCA is less feasible when using Gd-EOBDTPA. This is indeed correct and is due to the poorer reliability of the venous phase when using this contrast agent. However, clinical practice has taught us that the most important role of MRI is to differentiate HCA from FNH in young females using oral contraceptives. Whether a lesion is in fact an inflammatory HCA can provide useful additional information to predict b-catenin positivity (ie, b-cat-positive inflammatory HCAs), and malignant potential. ${ }^{9}$ Once radiological diagnosis confirms an inflammatory HCA, one could opt for biopsy to exclude b-catenin positivity, although current guidelines do not discuss this option at the present time. ${ }^{10}$

In conclusion, measuring lesion contrast uptake would be an attractive tool for differentiating HCAs and FNHs; however, we think the current status does not allow us to use this method in daily clinical practice.

${ }^{1}$ Department of Radiology, Erasmus MC University Medical Center, Rotterdam, The Netherlands

${ }^{2}$ Department of Radiology, Radboud University Medical Center, Nijmegen, The Netherlands

${ }^{3}$ Department of Gastroenterology and Hepatology, Erasmus MC University Medical Center, Rotterdam, The Netherlands

${ }^{4}$ Department of Surgery, Erasmus MC University Medical Center, Rotterdam, The Netherlands 


\section{References}

1. Vanhooymissen I, Thomeer MG, Braun LMM, et al. Intrapatient comparison of the hepatobiliary phase of Gd-BOPTA and Gd-EOB-DTPA in the differentiation of hepatocellular adenoma from focal nodular hyperplasia. J Magn Reson Imaging 2019;49(3):700-710.

2. Thomeer MG, Willemssen FE, Biermann KK, et al. MRI features of inflammatory hepatocellular adenomas on hepatocyte phase imaging with liver-specific contrast agents. J Magn Reson Imaging 2014;39(5): 1259-1264.

3. Reizine $\mathrm{E}$, Ronot $\mathrm{M}$, Pigneur F, et al. Iso- or hyperintensity of hepatocellular adenomas on hepatobiliary phase does not always correspond to hepatospecific contrast-agent uptake: Importance for tumor subtyping. Eur Radiol 2019;29(7):3791-3801.

4. van Aalten SM, Thomeer MG, Terkivatan T, et al. Hepatocellular adenomas: Correlation of MR imaging findings with pathologic subtype classification. Radiology 2011;261(1):172-181.

5. Yoneda N, Matsui $\mathrm{O}$, Kitao A, et al. Benign hepatocellular nodules Hepatobiliary phase of Gadoxetic acid-enhanced MR imaging based on molecular background. Radiographics 2016;36(7):2010-2027.

6. Sciarra A, Schmidt S, Pellegrinelli A, et al. OATPB1/B3 and MRP3 expression in hepatocellular adenoma predicts Gd-EOB-DTPA uptake and correlates with risk of malignancy. Liver Int 2019;39(1): 158-167.

7. Roux M, Pigneur F, Calderaro J, et al. Differentiation of focal nodular hyperplasia from hepatocellular adenoma: Role of the quantitative analysis of gadobenate dimeglumine-enhanced hepatobiliary phase MRI. J Magn Reson Imaging 2015;42(5):1249-1258.

8. Thomeer MG, Gest B, van Beek H, et al. Quantitative analysis of hepatocellular adenoma and focal nodular hyperplasia in the hepatobiliary phase: External validation of LLCER method using gadobenate dimeglumine as contrast agent. J Magn Reson Imaging 2018;47(3):860-861.

9. Nault JC, Couchy G, Balabaud C, et al. Molecular classification of hepatocellular adenoma associates with risk factors, bleeding, and malignant transformation. Gastroenterology 2017;152(4):880-894. e886.

10. European Association for the Study of the Liver. EASL clinical practice guidelines on the management of benign liver tumours. J Hepatol 2016;65(2):386-398.

DOI: 10.1002/jmri.27137

\section{Level of Evidence: 5 \\ Technical Efficacy Stage: 3}

